\title{
HEEL QUANTITATIVE ULTRASOUND PARAMETERS, SERUM 25-HYDROXYVITAMIN D AND PARATHYROID HORMONE LEVELS OF HEALTHY ADULT WOMEN IN GREECE
}

\author{
Efi V. Grigoriou ${ }^{1}$, Christos K. Kosmidis ${ }^{2 \star}$, George V. Dedoussis ${ }^{1}$, George P. Trovas ${ }^{2}$
}

1. Department of Dietetics - Nutrition, Harokopio University, 70 El. Venizelou St., 17671 Athens, Greece

2. Laboratory for Research of the Musculoskeletal System, School of Medicine, University of Athens, 10 Athinas St., 145 61, Athens, Greece.

*Correspondence to: chrikos1@otenet.gr

Objective: The objective of this observational cross-sectional study is to identify heel bone properties, specifically broadband ultrasound attenuation (BUA), speed of sound (SOS) and stiffness index (SI), in healthy Greek women, as well the relation of these parameters with age, serum 25-hydroxyvitamin D (25(OH)D) and parathyroid hormone (PTH).

Material and Methods: A population of 738 community dwelling women was recruited at the health promotion events carried out by the Hellenic Society for the Support of Patients with Osteoporosis in rural and urban areas throughout Greece. PTH and 25(OH)D were measured. Heel bone properties measured using quantitative ultrasound (QUS) device. The study was approved by the Ethics Committee of Harokopio University.

Results: The descriptive data of population are shown in the table 1 as mean \pm standard deviation. There is a significant difference of $B \cup A(114,05 \pm 15,7 \neq 107,66 \pm 15,7, p=0,039)$ and SOS $(1555,67 \pm 34,26 \neq 1506,0 \pm 18, \mathrm{p}=0,00)$ between normal $(15-65 \mathrm{pg} / \mathrm{mL})$ and high $(>65 \mathrm{pg} / \mathrm{mL})$ PTH level respectively. Sl and BUA are significantly different between all age groups as shown in table1 with $p=0,00$ for both parameters. $S 1$ is higher in $18-50$ age group in relation to $51-65$ and $>65$ age group ( $p=0,00$ and $p=0,03$ respectively).

Table 1: Descriptive characteristics

$\begin{array}{ll}\text { Total sample } & \mathbf{2 5}(\mathbf{O H}) \mathbf{D}(\mathbf{n g} / \mathbf{m L}) \\ \text { 18-50 years } & 19.87 \pm 7.57 \\ 51-65 \text { years } & 20.14 \pm 7,78 \\ >65 \text { years } & 19.36 \pm 7,66 \\ & 17.64 \pm 6,52\end{array}$

PTH $(\mathbf{p g} / \mathbf{m L})$
$39.67 \pm 14.75$
$36.05 \pm 14.15$
$43.29 \pm 14.85$
$47.29 \pm 16.03$

$\begin{array}{lll}\text { SI } & \text { BUA } & \text { SOS } \\ 89.95 \pm 18.38 & 113.78 \pm 15.67 & 1551.92 \pm 70.14 \\ 96.75 \pm 17.00 & 117.89 \pm 15.01 & 1560.13 \pm 88.95 \\ 84.51 \pm 16.7 & 110.12 \pm 14.6 & 1544.71 \pm 31.76 \\ 75.5 \pm 16.19 & 104.37 \pm 16 & 1528.91 \pm 29.00\end{array}$

Conclusions: The mean vitamin D levels of older Greek women (>50years) is below 20ng/mL, as well as BUA, SOS and SI are lower in older age groups (51-65 and >65). Given that low levels are associated with increased risk for fractures, this study highlights the emerging issue of 25(OH)D insufficiency in Greek women and the need for targeted interventions. All authors state that they have no conflicts of interest.

\begin{abstract}
Introduction
Osteoporosis is a major public health concern. Women have a higher risk of osteoporotic fracture compared with men ( 1 in 3 women and 1 in 5 men over 50 years). Quantitative bone ultrasound (QUS) is emerging as a low-cost, ionizing radiation-free, simple, and portable screening technique that is able to identify women at risk for the osteoporosis and that may be used by general practitioners in primary care. Lower values of the QUS parameters were associated with a significant increase of any subsequent fracture at any site. ${ }^{2}$ Vitamin $D$ deficiency is also among the most important clinical risk factors. Although recent data indicate that prevalence of vitamin $D$ deficiency can be common in countries previously considered as low risk (e.g Mediterranean countries) ${ }^{1}$, few data are available for Greek population. Low levels occur in elderly ${ }^{3}$, mothers after delivery ${ }^{4}$, children and adolescents ${ }^{5}$ and there are few data concerning adults 6 . Therefore, the evaluation of QUS parameters and vitamin D levels is of immense significance ${ }^{7}$. is an observational cross-sectional study that aims to identify the prevalence of vitamin D deficiency of healthy adults in Greece.
\end{abstract} Methods

A population of 738 community dwelling women was recruited at the health promotion events carried out by the Hellenic Society for the Support of Patients with Osteoporosis in rural and urban areas throughout Greece. Following a 12 hour fast, all subjects will have a blood drawn of venous blood used for serum isolation. Total calcium (Ca), phosphorus (P), parathyroid hormone $(\mathrm{PTH})$ and 25-hydroxyvitamin $\mathrm{D}(25(\mathrm{OH}) \mathrm{D})$ were measured. Heel bone properties measured using the Achilles quantitative ultrasound (QUS) device, a water-bath ultrasound system into which the subject places his heel. Achilles generates a band of frequencies from 200 to $600 \mathrm{kHz}$. It measures the broadband ultrasound attenuation (BUA), expressed in $\mathrm{dB} / \mathrm{MHz}$, and the speed of sound (SOS), expressed in $\mathrm{m} / \mathrm{s}$. The third variable, stiffness index (SI), is automatically calculated by Achilles from the BUA and the SOS, using the equation $\mathrm{SI}=\left(0.67^{\star} \mathrm{BUA}\right)+\left(0.28^{\star} \mathrm{SOS}\right)-420$. The SPSS statistical package used for the statistical analysis. The study was approved by the Ethics Committee of Harokopio University.

Results

The mean age of the population was $50.40 \pm 12.94$ years, (range, 20-86 years. The descriptive data of population are shown in the table 1 as mean \pm standard deviation. There is a significant difference of BUA $(114.05 \pm 15.7 \neq 107.66 \pm 15.7, \quad p=0.04)$ and SOS $(1555.67 \pm 34.26 \neq 1506.0 \pm 18.00 \mathrm{p}=0.00)$ between normal (15$65 \mathrm{pg} / \mathrm{mL}$ ) and high $(>65 \mathrm{pg} / \mathrm{mL}$ ) PTH level respectively. SI and BUA are significantly different between all age groups as shown in table1 with $p=0.00$ for both parameters. $S I$ is higher in 18-50 age group in relation to $51-65$ and $>65$ age group ( $p=0.00$ and $p=0.03$ respectively).

Table 1: Descriptive characteristics

$\begin{array}{llllll} & \text { 25(OH)D }(\mathbf{n g} / \mathrm{mL}) & \text { PTH }(\mathrm{pg} / \mathrm{mL}) & \text { SI } & \text { BUA } & \text { SOS } \\ \text { Total sample } & 19.87 \pm 7.57 & 39.67 \pm 14.75 & 89.95 \pm 18.38 & 113.78 \pm 15.67 & 1551.92 \pm 70.14 \\ \text { 18-50 years } & 20.14 \pm 7.78 & 36.05 \pm 14.15 & 96.75 \pm 17 & 117,89 \pm 15,01 & 1560.13 \pm 88.95 \\ 51-65 \text { years } & 19.36 \pm 7.66 & 43.29 \pm 14.85 & 84.51 \pm 16.7 & 110,12 \pm 14,6 & 1544.71 \pm 31.76 \\ >65 \text { years } & 17.64 \pm 6.52 & 47.29 \pm 16.03 & 75.5 \pm 16.19 & 104,37 \pm 16 & 1528.91 \pm 29\end{array}$

\section{Conclusions}

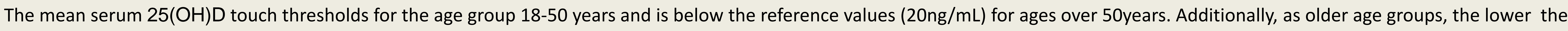
levels of QUS parameters (BUA, SOS and SI) are lower in older age groups (51-65 and >65). BUA and SOS values are significant higher in subjects with normal PTH (15-65pg/mL), than those with high levels $(>65 \mathrm{pg} / \mathrm{mL})$. Low levels of $25(\mathrm{OH}) \mathrm{D}$ and QUS parameters, are associated with increased risk for fractures and exacerbate bone loss. ${ }^{1,2}$ Beyond the skeletal consequences, low serum vitamin D, has numerous nonkeletal effects ${ }^{8}$. This study highlights the emerging issue of $25(\mathrm{OH}) \mathrm{D}$ insufficiency and the decrease of BUA, SOS and SI at older ages, in Greek population, that should get priority in public health strategies and the need for targeted interventions.

\section{Acknowledgements}

The study was supported by the Hellenic Society for the Study of Bone Metabolism

\section{References}

1. Holick MF. Vitamin D Deficiency. N Engl J Med 2007;357:266-281. 2. Marin F. et al. Relationship Between Bone Quantitative Ultrasound and Fractures: A Meta-Analysis. J Bone Miner Res 2006;21:1457-1463 3. Papapetrou PD, et al. Vitamin D deficiency in the elderly in Athens, Greece. Bone Miner Metab 2007;25:198-203. 4. Nicolaidou P, et al. Low Vitamin D Status in Mother-Newborn Pairs in Greece. Calcif Tissue Int 2006;78:337-342.. 5. Lapatsanis D, et al. Vitamin D: A Necessity for Children and Adolescents in Greece. Calcif Tissue Int 2005;77:348-355. 6. Pazaitou-Panayiotou K, et al. Height, whole Body Surface Area, gender, working outdoors, and sunbathing in previous summer are important determinants of serum 25-hydroxyvitamin D levels. Exp Clin Endocrinol Diabetes. 2012;120(1):14-22. 7. Dusso AS, et al. Vitamin D. Am J Physiol Renal Physiol 2005;289:F8-F28. 8. Grober U, et al. Vitamin D: Update 2013 From rickets prophylaxis to general preventive healthcare. Dermatoendocrinol. 2013;5(3):331-347 